\title{
CORRIGENDUM
}

\section{The size and complexity of dolphin brains - a paradox? - CORRIGENDUM}

\author{
S. HUGGENBERGER
}

doi:10.1017/So025315408000738, Published by Cambridge University Press, September 2008.

The author has identified a miscalculation in Figure 2 in the study of Huggenberger (2008), on page 1105. The value for the chimpanzee brain in the left graph of Figure 2 must be $14.4^{\star} 10^{6}$ neocortical neurons per gram brain weight (not $1.44^{\star} 10^{6} / \mathrm{g}$ ). Therefore, on page 1104, the two last sentences in this study should read 'Accordingly, the dolphins have more neocortical neurons in relation to their brain weight than the elephant but less than the Rhesus monkey (Figure 2). The hedgehog and the rat have approximately the same number of neocortical neurons per gram brain weight as the human which is only surpassed by the cat and the chimpanzee.'

\section{REFERENCE}

Huggenberger S. (2008) The size and complexity of dolphin brains - a paradox? Journal of the Marine Biological Association of the United Kingdom 88, 1103-1108. 